\title{
Applying Data Envelopment Analysis Principle in Ordinal Multi Criteria Decision Analysis
}

\author{
Moshe Kress $^{1}$
}

\begin{abstract}
We consider a multicriteria decision analysis (MCDA) problem where importance of criteria, and evaluations of alternatives with respect to the criteria, are expressed on a qualitative ordinal scale. Using the extreme-point principle of Data Envelopment Analysis (DEA), we develop a two-parameter method for obtaining overall ratings of the alternatives when preferences and evaluations are made on an ordinal scale. We assume no parametric setup other than the two parameters that reflect minimum intensities of discriminating among rank positions: one parameter for the alternatives' ranking and one for the criteria ranking. These parameters are bounded by the ordinal input data, and they imply a universal tie among the alternatives when both parameters are selected to be zero. We describe the model, discuss its theoretical underpinning, and demonstrate its application.
\end{abstract}

Keywords: Multiple criteria analysis, ordinal scale, DEA, extreme-point, discriminating factor.

\section{Introduction}

We consider an ordinal multi-criteria decision problem where all inputs - weights of criteria and evaluations of alternatives with respect to the criteria - are given in terms of qualitative ordinal rankings or ordinal scores such as the Likert scale [17], [2]. Ordinal elicitations of assessments and preferences in an MCDA setting are less cognitively demanding than cardinal ones [16]. In a decision environment that may be uncertain and nebulous, a simple ordering is more natural to express preferences than cardinal values [8], [15]. Therefore, it may be easier to implement an ordinal preference setting, rather than cardinal, in complex multi-criteria problems. The question is how to aggregate the ordinal input into an overall evaluation that can prioritize the alternatives in a reasonable and consistent way.

The main thrust of this note is to propose a useful, transparent and easily-implementable method for assessing multicriteria linear value-functions in a purely ordinal setting. The

\footnotetext{
${ }^{1}$ Operations Research Department, Naval Postgraduate School, Monterey, California 93943, e-mail: mkress@nps.edu
} 
method has the following two main features: (1) It is founded on the solid theoretical ground of the well-established Data Envelopment Analysis (DEA) methodology [5], (2) Besides two parameters, controlled by the decision-maker and functioning as "discriminating rheostat", the method is fully ordinal and does not rely on any cardinal input, including no criteria weights.

There have been several attempts to address a variant of this problem where the objective is to elicit cardinal weights of criteria from their ordinal ranking. Methods range from procedures [1] and models \{12], [13] converting qualitative (linguistic) terms into cardinal or fuzzy settings, which are essentially equivalent to quantitative approaches, to theoretical approaches based on partial preorder of reference alternatives whose values are known, as well as from other partial preorders [18], to a transformation, called global outranking method, from the two dimensional (alternative, criterion) space into a single dimensional ranking of the alternatives [21]. Other approaches for dealing with ordinal data in an MCDA setting are reported, among others, in [4], [11], [14], [19], [23], and [24]. Recent contributions to the topic address non-uniform scales, which are treated using the concept of ordinal proximity measure [9], [10]. Non-uniform scales are implicitly present in our approach in this paper, as shown later on.

The main thrust of this note is to propose a useful, transparent and easily-implementable method for assessing multicriteria linear value-functions in a purely ordinal setting. The method has the following two main features: (1) It is founded on the solid theoretical ground of the well-established Data Envelopment Analysis (DEA) methodology [5], (2) Besides two parameters, controlled by the decision-maker and functioning as "discriminating rheostat", the method is fully ordinal and does not rely on any cardinal input, including no criteria weights.

The method is based on an extreme-point principle and models developed in [6] and [7]. The models are MCDA applications of DEA [5], which is a widely-accepted method for measuring efficiencies of decision making units in a most equitable way. The multiple parameters in [6] and the non-linearity in [7] are reduced here to a two-parameter linear optimization model, which is transparent to and easily understandable by decision makers. The model is also easily implementable for real-world problems on a spreadsheet. An important feature highlighted here is the sensitivity of the decision outcome to the two parameters called discrininating factors, which represent the decision-maker's general strength of opinion or preferences.

Section 2 describes the model and Section 3 presents an example and short analysis. Concluding remarks are given in Section 4.

\section{Model}

In many legacy MCDA models (e.g., the Analytic Hierarchy Process (AHP) [22]) the setup is rather simple: each of $m$ criteria is assigned a weight $w_{i}, i=1, \ldots, m$, and each alternative $j, j=1, \ldots, n$, is evaluated with respect to each criterion $i$ and is given a value $v_{i j}$. The overall evaluation $V_{i}$ of alternative $i$ is the weighted sum of its values, that is, $V_{j}=\sum_{i=1}^{m} V_{i j}$, where $V_{i j}=w_{i} v_{i j}$. In the purely ordinal setting this type of evaluations are not applicable anymore. If 
we only know that, say, $w_{1}>w_{2}>\ldots>w_{m}$ and that, for some criterion $i, v_{i 1}>v_{i 2}>\ldots>v_{i n}$, what can we say about the $V_{i j}$ svalues and ultimately about the alternatives' final ratings $V_{j}, j=1, \ldots, n$ ?

Suppose that on each criterion, the $n$ alternatives are evaluated on an ordinal scale having $r$ rank positions. For example, if $r=5$ then we have the Likert scale (Likert, 1932; Allen and Seaman, 2007). Similarly, the importance or weight of each of the $m$ criteria is determined on an ordinal scale having $s$ rank positions. The choices of the ordinal scales - the parameters $r$ and $s-$ are completely arbitrary. For example, the alternatives and criteria could be strictly ordered on $n$ and $m$ rank positions, respectively. Higher rank (lower index number) indicates higher value for an alternative and higher importance (weight) for a criterion. Let $x_{i j}$ denote the "value" of being ranked in the $j-t h$ rank position with respect to a criterion ranked in the $i$-thrank position. We make no specific requirements regarding the values $x_{i j}$ other than the following natural assumptions:

(1) The values are nonnegative, $x_{i j} \geq 0$.

(2) A higher rank of an alternative on a certain criterion has a larger value than a lower rank on that criterion,

$$
x_{i j}-x_{i, j+1}>0, i=1, \ldots, s, j=1, \ldots, r-1 .
$$

The third assumption relates to the rank positions of the criteria. The effect of the ordering of the criteria leads to two possible interpretations: a strict one and a relaxed one. In the strict interpretation we require that an alternative's rank position on a higher ranked criterion has a lager value $x_{i j}$ than the same rank position on a lower ranked criterion. That is,

$$
x_{i j}-x_{i+1, j}>0, i=1, \ldots, s-1, j=1, \ldots, r .
$$

The logic behind these relations is that being, say, best on a more important criterion carries more value (larger $V_{i j}$ value) than rated best on a less important criterion. A more relaxed assumption is simply to require that the total value assigned to a higher ranked criterion is larger than the total value of a lower ranked criterion, that is

$$
\sum_{j=1}^{r}\left(x_{i j}-x_{i+1, j}\right)>0, i=1, \ldots, s-1 .
$$

We will analyze and compare the two aforementioned interpretations of criteria rankings.

Next, we define, 


$$
\begin{gathered}
a_{j}^{k}(l)=\left\{\begin{array}{lr}
1 & \text { if alternative } k \text { is ranked } j-t h \text { on criterion } l \\
0 & \text { otherwise }
\end{array}\right. \\
c_{i}^{l}=\left\{\begin{array}{lr}
1 & \text { if criterion } l \text { is ranked in the } i-t h \text { position } \\
0 & \text { otherwise }
\end{array}\right.
\end{gathered}
$$

If the $x_{i j}$ values were known, then the overall evaluation, or rating, of alternative $k$ would be:

$$
V_{k}=\sum_{l=1}^{m} \sum_{i=1}^{s} \sum_{j=1}^{r} a_{j}^{k}(l) c_{i}^{l} x_{i j}, \quad k=1, \ldots, n .
$$

Similarly to many other MCDM models (e.g., [22]) the alternative with the highest $V$ value is the most preferred. The question is how to determine the $x_{i j}$ values in a reasonable and "fair" way, consistent with equations (1) and (2) (or, alternatively, (2a)).

To do so, we propose an extreme-point principle, which is well established in the theory of Data Envelopment Analysis (DEA) [5]. The idea is to give each alternative its "best shot" for presenting itself. In other words, each alternative can select its own $x_{i j}$ values such that it would get the highest possible rating subject to the constraints (1) and (2) (or (2a)), and the requirement that when the selected $x_{i j}$ values are applied to the other alternatives, no alternative gets a rating higher than a certain bound which is normalized to be equal to 1 . This is an "equitable" concept in the sense that the rating of each alternative is evaluated in exactly the same way as the others'.

To put this idea into practice, we define two discriminating factors. Similarly to the idea in (Punkka and Salo 2013) the discriminating factors express how clearly a decision-maker can distinguish among rank positions of the alternatives and the criteria. These factors are related to the concept of proximity measure described in [9] and [10], and are somewhat similar to the concept of value-difference in [20]. Define $\varepsilon$ as the discriminating factor for the alternatives rank position. This is the minimum gap between two consecutive rank positions of alternatives. Formally,

$$
x_{i j}-x_{i, j+1} \geq \varepsilon, i=1, \ldots, s, j=1, \ldots, r-1 .
$$

Similarly, we define $\delta$ as the discriminating factor for the criteria. That is,

$$
x_{i j}-x_{i+1, j} \geq \delta, i=1, \ldots, s-1, j=1, \ldots, r
$$

for the strict case, and

$$
\sum_{j=1}^{r}\left(x_{i j}-x_{i+1, j}\right)>\delta, i=1, \ldots, s-1 .
$$

for the relaxed case. 
As in standard DEA (Primal) model, we solve $n$ linear programming problems - one for each alternative. As a convention for simplifying notation, an alternative for which the rating $V$ is computed, and therefore appears in the objective function, we assign the index 0 . Thus, we solve the following $n$ LP problems:

$$
\begin{aligned}
& \operatorname{Max} V_{0}=\operatorname{Max} \sum_{l=1}^{m} \sum_{i=1}^{s} \sum_{j=1}^{r} a_{j}^{0}(l) c_{i}^{l} x_{i j} \\
& \text { s.t. } \\
& \sum_{l=1}^{m} \sum_{i=1}^{s} \sum_{j=1}^{r} a_{j}^{k}(l) c_{i}^{l} x_{i j} \leq 1, k=1, \ldots, n
\end{aligned}
$$

Constraints (6) and (7) (or (7a))

$$
x_{i j} \geq 0
$$

In the DEA context, alternatives for which the objective in (8) reaches the value 1 are said to be efficient. In our MCDA context we call such alternative non-dominated. Alternatives for which the corresponding functional in (8) is less than 1 are dominated by those alternatives for which the functional is equal 1. Based on the ordinal evaluations, the objective of the decision maker is to narrow down the set of non-dominated alternatives and thus identify the desired alternatives. If $\varepsilon=0$ then all the alternatives are trivially nondominated as they all can reach the highest rating of 1 . In that case each alternative can set $x_{i j}=\frac{1}{m}, i=1, \ldots, s, j=1, \ldots, r$, and all ratings $V_{k}, k=1, \ldots, n$, become the same at 1 . As the values of $\varepsilon$ and $\delta$ increase from 0 , alternatives begin to separate in their values; the better alternatives maintain the rating 1 but the other alternatives start to get values $V$ lower than 1 . This process ends when either $\varepsilon$ or $\delta$ become too large so that problem (8)-(11) becomes infeasible. For example, if $\varepsilon=\delta$ then the maximum possible value of $\varepsilon$ is obtained when it is taken as a decision variable in (8) - (11), and we add the term $M \varepsilon$ to the objective function, where $M$ is a large constant. Formally, 


$$
\begin{aligned}
& \operatorname{Max} \sum_{l=1}^{m} \sum_{i=1}^{s} \sum_{j=1}^{r} a_{j}^{0}(l) c_{i}^{l} x_{i j}+M \varepsilon \\
& \text { s.t. } \\
& \sum_{l=1}^{m} \sum_{i=1}^{s} \sum_{j=1}^{r} a_{j}^{k}(l) c_{i}^{l} x_{i j} \leq 1, \quad k=1, \ldots, n
\end{aligned}
$$

$$
\varepsilon, x_{i j} \geq 0 \text {. }
$$

\section{Example and Analysis}

To demonstrate the model and its features, and analyze its outcomes, consider the following example with $n=6$ alternatives and $m=4$ criteria. The value of the alternatives with respect to the criteria, and the importance of the criteria are evaluated on a Likert scale, that is, $r=s=5$. To be consistent with the notation thus far, we reverse the conventional definition of the scale and assign the following rank values for an alternative and a criterion, respectively:

1 - Most Valuable/Important,

2 - Very Valuable/Important,

3 - Valuable/ Important,

4 - Rather Valuable/ Important,

5 - Marginally Valuable/Important.

Table 1 presents the Likert scores for the criteria.

Table 1. Likert Scores for Criteria Importance

\begin{tabular}{|c|c|}
\hline Criterion & $\begin{array}{l}\text { Likert } \\
\text { Score }\end{array}$ \\
\hline $\mathrm{C} 1$ & 2 \\
\hline $\mathrm{C} 2$ & 1 \\
\hline $\mathrm{C} 3$ & 4 \\
\hline $\mathrm{C} 4$ & 5 \\
\hline
\end{tabular}


Table 2 presents the Likert scores for the six alternatives, labeled A1,..,A6, with respect to the four criteria. Notice that no alternative is trivially dominated by another, that is, no alternative is ranked lower than another alternative on all criteria. Therefore, there is no obvious inferior alternative that we could discard from further consideration.

First, we consider the strict case where we use constraints (7) in problem (8) - (11). Table 3 presents the non-dominated alternatives whose functional (8) reaches the value 1 and therefore they are most preferred. We can see that as the discriminating factors $\varepsilon$ and $\delta$ increase, fewer alternatives stay non-dominated. Also notice that, as discussed above, when $\varepsilon=0$, which practically means that the decision maker has no clear way of distinguishing among the alternatives - all alternatives are equally valuable. Table 3 also displays the range of discriminating factors for which problem (8) - (11) (with constraints (7)) is feasible.

Table 2. Likert Scores for Alternatives' Values with Respect to the Four Criteria.

\begin{tabular}{|c|c|c|c|c|c|c|}
\hline & A1 & A2 & A3 & A4 & A5 & A6 \\
\hline C1 & 2 & 4 & 3 & 2 & 2 & 3 \\
\hline C2 & 3 & 2 & 5 & 3 & 2 & 1 \\
\hline C3 & 3 & 2 & 1 & 5 & 1 & 4 \\
\hline C4 & 2 & 2 & 2 & 1 & 3 & 5 \\
\hline
\end{tabular}

Table 3: Non-Dominated Alternatives for the Case of Strict Criteria Discrimination

\begin{tabular}{|c|c|c|c|c|c|c|}
\hline & \multicolumn{7}{|c|}{$\varepsilon$} \\
\hline \multirow{2}{*}{$\delta$} & $\mathrm{A} 1, \ldots, \mathrm{A} 6$ & $\mathrm{~A} 1, \ldots, \mathrm{A} 6$ & $\mathrm{~A} 1, \ldots, \mathrm{A} 6$ & $\mathrm{~A} 5, \mathrm{~A} 6$ & Infeasible \\
\hline & .02 & $\mathrm{~A} 1, \ldots, \mathrm{A} 6$ & $\mathrm{~A} 1, \ldots, \mathrm{A} 6$ & $\mathrm{~A} 1, \ldots, \mathrm{A} 6$ & $\mathrm{~A} 5, \mathrm{~A} 6$ & Infeasible \\
\cline { 2 - 7 } & .04 & $\mathrm{~A} 1, \ldots, \mathrm{A} 6$ & $\mathrm{~A} 1, \ldots, \mathrm{A} 6$ & $\mathrm{~A} 5, \mathrm{~A} 6$ & Infeasible & Infeasible \\
\cline { 2 - 7 } & .06 & $\mathrm{~A} 1, \ldots, \mathrm{A} 6$ & $\mathrm{~A} 1, \ldots, \mathrm{A} 6$ & $\mathrm{~A} 5, \mathrm{~A} 6$ & Infeasible & Infeasible \\
\hline & .08 & $\mathrm{~A} 1, \ldots, \mathrm{A} 6$ & $\mathrm{~A} 1, \mathrm{~A} 2, \mathrm{~A} 5, \mathrm{~A} 6$ & Infeasible & Infeasible & Infeasible \\
\hline & .1 & $\mathrm{~A} 1, \ldots, \mathrm{A} 6$ & Infeasible & Infeasible & Infeasible & Infeasible \\
\hline
\end{tabular}

Alternatives A5 and A6 remain non-dominated throughout. No feasible combination of the discriminating factors can bring any of these two alternatives to an objective value smaller than 1. The conclusion is that, based on the Likert scores provided for the importance of the alternatives, and the values of the alternatives with respect to the criteria, the two alternatives, A5 and A6, are equally desired at the top. 
Table 4 presents the $\mathrm{V}$ values for the six alternatives when we assume that $\varepsilon=\delta$. In that case, the maximum feasible value of the common discriminating parameter is 0.05 . We can see now in detail the general picture presented in Table 3. First, if the common discriminating factor is less than or equal 0.03, all six alternatives are non-dominated; the decision maker cannot identify a smaller set of desired alternatives. For discriminating factor greater than or equal .04 we see that the six alternatives are divided into 3 subsets: the non-dominated alternatives A5 and A6, the second tier of alternatives: A1 and A2, and the inferior alternatives: A3 and A4. These observations are consistent with the results in Table 3.

Table 4: $V$ Scores of Alternatives for Equal Discriminating Factors

\begin{tabular}{|c|c|c|c|c|c|c|}
\hline \multirow{2}{*}{$\varepsilon=\delta$} & \multicolumn{7}{|c|}{ Alternatives } \\
\cline { 2 - 7 } & $\mathrm{A} 1$ & $\mathrm{~A} 2$ & $\mathrm{~A} 3$ & $\mathrm{~A} 4$ & $\mathrm{~A} 5$ & $\mathrm{~A} 6$ \\
\hline 0 & 1 & 1 & 1 & 1 & 1 & 1 \\
\hline .01 & 1 & 1 & 1 & 1 & 1 & 1 \\
\hline .02 & 1 & 1 & 1 & 1 & 1 & 1 \\
\hline .03 & 1 & 1 & 1 & 1 & 1 & 1 \\
\hline .04 & .99 & .99 & .95 & .95 & 1 & 1 \\
\hline .05 & .90 & .90 & .85 & .85 & 1 & 1 \\
\hline
\end{tabular}

Table 5, which is similar to Table 3, presents the non-dominated alternatives for the relaxed case where constraints (7a) replace constraints (7) in problem (8) - (11). Notice that in this case the significant values of $\delta$ are order of magnitude larger than those values in the strict case, which is reasonable considering we compare now sums of rank values.

Table 5: Non-Dominated Alternatives for the Case of Relaxed Criteria Discrimination

\begin{tabular}{|c|c|c|c|c|c|c|}
\hline & \multicolumn{7}{|c|}{$\varepsilon$} \\
\hline \multirow{5}{*}{$\delta$} & & 0 & .02 & .04 & .06 & .08 \\
\cline { 2 - 7 } & 0 & $\mathrm{~A} 1, \ldots, \mathrm{A} 6$ & $\mathrm{~A} 1, \ldots, \mathrm{A} 6$ & $\mathrm{~A} 1, \ldots, \mathrm{A} 6$ & $\mathrm{~A} 1, \ldots, \mathrm{A} 6$ & $\mathrm{~A} 5, \mathrm{~A} 6$ \\
\cline { 2 - 7 } & .1 & $\mathrm{~A} 1, \ldots, \mathrm{A} 6$ & $\mathrm{~A} 1, \ldots, \mathrm{A} 6$ & $\mathrm{~A} 1, \ldots, \mathrm{A} 6$ & $\mathrm{~A} 1, \ldots, \mathrm{A} 6$ & $\mathrm{~A} 5, \mathrm{~A} 6$ \\
\cline { 2 - 7 } & .2 & $\mathrm{~A} 1, \ldots, \mathrm{A} 6$ & $\mathrm{~A} 1, \ldots, \mathrm{A} 6$ & $\mathrm{~A} 1, \ldots, \mathrm{A} 6$ & $\mathrm{~A} 1, \mathrm{~A} 2, \mathrm{~A} 4, \mathrm{~A} 5, \mathrm{~A} 6$ & Infeasible \\
\cline { 2 - 7 } & .3 & $\mathrm{~A} 1, \ldots, \mathrm{A} 6$ & $\mathrm{~A} 1, \ldots, \mathrm{A} 6$ & $\mathrm{~A} 1, \ldots, \mathrm{A} 6$ & $\mathrm{~A} 5, \mathrm{~A} 6$ & Infeasible \\
\cline { 2 - 7 } & .4 & $\mathrm{~A} 1, \ldots, \mathrm{A} 6$ & $\mathrm{~A} 1, \ldots, \mathrm{A} 6$ & $\mathrm{~A} 1, \ldots, \mathrm{A} 6$ & Infeasible & Infeasible \\
\hline & .5 & $\mathrm{~A} 1, \ldots, \mathrm{A} 6$ & $\mathrm{~A} 1, \ldots, \mathrm{A} 6$ & $\mathrm{~A} 1, \mathrm{~A} 2, \mathrm{~A} 4, \mathrm{~A} 5, \mathrm{~A} 6$ & Infeasible & Infeasible \\
\cline { 2 - 7 } & .55 & $\mathrm{~A} 1, \ldots, \mathrm{A} 6$ & $\mathrm{~A} 1, \ldots, \mathrm{A} 6$ & $\mathrm{~A} 5, \mathrm{~A} 6$ & Infeasible & Infeasible \\
\cline { 2 - 7 } & .6 & $\mathrm{~A} 1, \ldots, \mathrm{A} 6$ & $\mathrm{~A} 1, \ldots, \mathrm{A} 6$ & Infeasible & Infeasible & Infeasible \\
\cline { 2 - 7 } & .7 & $\mathrm{~A} 1, \ldots, \mathrm{A} 6$ & Infeasible & Infeasible & Infeasible & Infeasible \\
\hline
\end{tabular}


.8

Infeasible $\quad$ Infeasible

Infeasible

Infeasible

Infeasible

As one can see, the results of the relaxed model are consistent with those of the strict model presented in Table 3. If we assume that is $\varepsilon=0.1 \delta$ then the maximum feasible discriminating factor is $\delta=.476$, in which case both alternatives A5 and A6 are still at the top with $V_{A 5}=V_{A 6}=1$. For the other four alternatives, we have, $V_{A 1}=V_{A 2}=.91$ and $V_{A 3}=V_{A 4}=.86$, which is consistent with the results in Table 4, for the strict discrimination case. We conclude that for the two types of the criteria weights' discriminating factors - the strict and the relaxed - the results are consistent: alternatives A5 and A6 are indistinguishable and ranked at the top. The set of non-dominated alternatives expands as the decision maker is less convinced about discriminating among rank positions.

\section{Conclusions}

We presented a simple, equitable and intuitive method for evaluating MCDA problems with ordinal inputs. The method has a theoretical foundation derived from extreme-point principle manifested in Data Envelopment Analysis. Two parameters - one associated with the criteria and one with the alternatives - called discriminating factors, play a role of lower bounds on gaps among rank positions. Higher lower bounds indicate stronger preferences among rank positions. Low values of the discriminating factors, which indicates weak preferences and/or opinions, result in more tied alternatives at the top rating, as one would expect. As the discriminating factors increase better alternatives are distinguished from the other. The methodology presented is flexible enough to accommodate variability among criteria where the decision maker may be more confident about his preferences on one criterion than on another. In our model such a situation would be manifested by a vector of $\varepsilon$ values - one for each criterion - rather than a single factor. We also note that the proposed model can accommodate additional, not necessarily ordinal, requirements and assumptions regarding the criteria and the alternatives. For example, if the weight of criterion $\mathrm{C} 2$ in the example in Section 3 is evaluated to be at least two times the weight of $\mathrm{C} 1$, then problem (8) - (11) is modified by adding the constraint $\sum_{j=1}^{6} x_{2 j}-2 \sum_{j=1}^{6} x_{1 j} \geq 0$.

\section{References}

[1] Alfares H. K. and Duffuaa, S. O. 2009. Assigning Cardinal Weights in MultiCriteria Decision Making Based on Ordinal Ranking. J. Multi-Crit. Decis. Anal. 15: $125-133$.

[2] Allen, I. E. and Seaman C. A. 2007. Likert scale and data analyses. Quality Progress 64-65. 
[3] Atwater, B., and Uzdzinski, J. 2014. Wholistic Sustainment Maturity: The Extension of System Readiness Methodology across all Phasesof the Lifecycle of a Complex System. Procedia Computer Science 28, 601-609.

[4] Belton, V. and Stewart, T. J. 2001. Multiple Criteria Decision Analysis: An Integreated Approach. Dordrecht: Kluwer Academic Publishing.

[5] Charnes, A., Cooper, W. W. and Rhodes, E. 1978. Measuring the efficiency of decision making units. European Journal of Operational Research 2: 429-444.

[6] Cook, W. D. and Kress, M. 1991. A multiple criteria decision model with ordinal preference data. European Journal of Operational Research 54: 191-198.

[7] Cook, W. D. and Kress, M. 1996. An extreme-point approach for obtaining weighted ratings in qualitative multicriteria decision making. Naval Research Logistics 43: 519-531.

[8] Fasolo, B. and Bana e Costa, C. A. 2014. Tailoring Value Elicitation to Decision Makers' Numeracy and Fluency: Expressing Value Judgments in Numbers or Words. Omega 44: 83-90.

[9] Garcia-Lapresta, J. L. and Gonzales del Pozo, R. 2019. An Ordinal Multi-Criteria Decision-Making Procedure Under Imprecise Linguistic Assessments. European Journal of Operational Research 279 (1): 159-167.

[10] Garcia-Lapresta, J. L. and Perez-Roman, D. 2018. Aggregating Opinions in NonUniform Ordered Qualitative Scales. Applied Soft Computing 67: 652-657.

[11] Gomes, L. F. A. M., Mury, A. R. and Gomes, C. F. S. 1997. Multicriteria ranking with ordinal data. Systems Analysis Modelling Simulation 27 (2-3): 139-145.

[12]Herrera, F., Herrera-Viedma, E. and Martinez, L. 2008. A Fuzzy Linguistic Methodology to Deal with Unbalanced Linguistic Term Sets. IEEE Transactions on Fuzzy Systems 16: 354-370.

[13]Herrera-Viedma, E. and Lopez-Herrera, A. G. 2007. A Model of an Information Retrieval System with Unbalanced Fuzzy Linguistic Information. International Journal of Intelligent Systems 22: 1197-1214.

[14]Koksalan, M., Karwan, M. H. and Zionts, S. 1988. An approach for solving discrete alternative multiple criteria problems involving ordinal data. Naval Research Logistics Quarterly 35: 625-642.

[15]Larichev, O. I., and Brown, R. 2000. Numerical and Verbal Decision Analysis: Comparison on Practical Cases. Journal of Multi-Criteria Decision Analysis 9: 263274.

[16]Larichev, O. I. 1992. Cognitive validity in design of decision-aiding techniques. Journal of Multi-Criteria Decision Analysis 1 (3): 127-138.

[17]Likert, R. 1932. A Technique for the Measure of Attitudes. Archives of Psychology 22 (140): 1-55. 
[18] Marichal, J-L and Roubens, M. 2000. Determination of weights of interacting criteria from a reference set. European Journal of Operational Research 124 (3): 641-650.

[19] Moshkovich, H.M., Mechitov, A.I., Olson, D.L. 2002. Ordinal judgments in multiattribute decision analysis. European Journal of Operational Research 137 625641.

[20]Punkka, A., and Salo, A. 2013. Preference Programming with incomplete ordinal information. European Journal of Operational Research 231 141-150.

[21]Roubens, M. 1982. Preference relations on actions and criteria in multicriteria decision making. European Journal of Operational Research 10: 51-55.

[22] Saaty, T. L. 1980. The Analytic Hierarchy Process. New York: McGrow Hill.

[23] Salo, A. and Punkka, A. 2005. Rank inclusion in criteria hierarchies. European Journal of Operational Research 163 (2): 338-356.

[24]Xu, X. 2001. A multiple criteria ranking procedure based on distance between partial preorders. European Journal of Operational Research 133 (1): 69-80.

Received 18.02.2021, Accepted 17.03.2021 